\title{
Design of Stepper Motor Control Circuit with Adjustable Torque
}

\section{Lin Fang ${ }^{1, \mathrm{a}}$, Wang $\mathrm{Yu}^{2 *, \mathrm{~b}}$, Fu Yi-bin ${ }^{2, \mathrm{c}}$, Lu Fan ${ }^{2, \mathrm{~d}}$, Chang Zhen ${ }^{1,2, \mathrm{e}}$, Qiu Xiao-han ${ }^{1,2, f}$}

${ }^{1}$ University of Science and Technology of China, Hefei 230031, China;

${ }^{2}$ Anhui Institute of Optics and Fine Mechanics, Chinese Academy of Science, Hefei 230031, China.

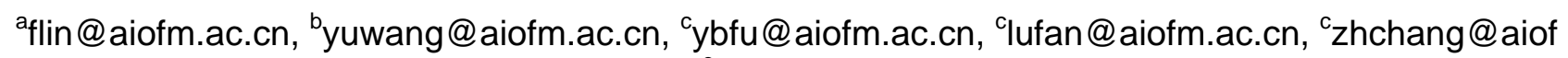
m.ac.cn, ${ }^{c} x$ hqu @aiofm.ac.cn

Keywords: stepper motors; PWM control; light-shifting devices; driving torque

\begin{abstract}
A design of a circuit controlling stepper motors in light-shifting device of the satellite-born spectrometer was proposed. This design utilizes FPGA as the core control chip and LMD18200 as the driving chip of stepper motors. To reduce the abrasion of bearings and overcome the increase of the resistance in orbit operation, a function that the rotation speed and the driving torque are able to be adjusted is added into this design. PWM control is implemented to adjust driving torque. The real tests verified that the design's range of controlling driving torque and rotation speed match the requests of the spectrometer.
\end{abstract}

\section{Introduction}

A stepper motor is a brushless electric motor that changes digital pulses into angular displacement and linear displacement. Stepper motors have the characteristics of low inertia, high positioning accuracy, no accumulate error, self lock, simple control and etc. Stepper motors are widely used in medical instruments, precision machineries, industrial control systems and space equipments.

However, stepper motors have a disadvantage, that it rotates in a step mode but not in constant speed. That makes the load, driven by the motor, move un-smoothly and have acceleration and deceleration process in each step. This process can be explained as following. The rotation speed of a stepper motor is decided by the frequency of the driving clock, and each clock the motor moves one step. If the driving torque, generated by electric current, is larger than adequate, it pushes the wheel from one position to next position earlier. Before next driving clock arrived, the wheel will be held in the position as the electric current hasn't been deserted and liberate slightly as the current is not fixed. Also, when the wheel is held, as the voltage is still on the winding, the current should be increased quickly and make the motor create further thermal consumption. On the other hand, if the driving torque is less than adequate, the motor would not be able to move or lose steps. Therefore, a proper driving torque, usually decided by the winding current, can reduce the liberation and thermal consumption, also assure the proper rotation. In most applications, designers can select a proper driving torque according to a certain speed. However, if the speed or the required torque changes, a fixed driving torque would not be always suitable.

In the real project, a satellite-born spectrometer ${ }^{[1]}$ incorporates two stepper motors to switch light paths $^{[2]}$. The diagram of the light-shifting device is shown in Fig.1. There is a stepper motor whose shaft is connected to a turntable. An optical lens is fixed on the turntable and rotates with it. A permanent magnet is attached on the side of the turntable. When the magnet comes in the close proximity to the hall effect proximity switch, an electronic signal is generated and delivered to the diver circuit. The circuit determines the position " 0 " of the stepper motors depending on receiving their hall signal. The stepper motor rotates in one direction and calibrates position " 0 ” to eliminate the error caused by out of step. A two-phase four-wire hybrid stepper motor with step angle $0.9^{\circ}$ was selected and used in the light-shifting device. 


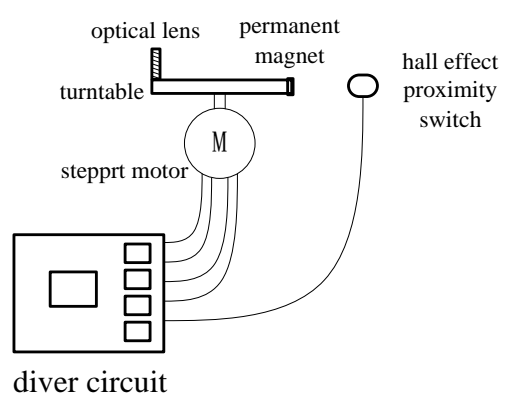

Fig.1 The diagram of the light-shifting device

Since the spectrometer needs to work for a long term (8 years), to reduce the abrasion of bearings, these two stepper motors need to be driven with a proper driving torque. Furthermore, due to the increase of the resistance after the lunching of the satellite and the device has been working for a long period, an adjustable driving torque is required.

In this application, PWM(pulse width modulation) is implemented to adjust the winding current and driving torque of the stepper motors. An FPGA is selected to generate driving clock and PWM pulses. With the adjustment of the frequency of the driving clock and the duty-ratio of the PWM pulses, the rotation speed and the driving torque are both able to be adjusted.

To avoid repeating development, two stepper motor controllers use same design. Thus, the design should have a wider adjustment range of the rotation speed and the driving torque. Furthermore, to reuse this design in other similar applications, the adjustment range is designed further larger. The design specifications of this controller are listed below.

1.This controller has an RS-422 UART port to communicate with a host CPU.

2.This controller is able to change the speed from $0.6 \mathrm{~s} /$ circle $-8 \mathrm{~s} /$ circle.

3.This controller is able to change the duty-ratio from $0-100 \%$ divided into 128 shifts of the PWM.

\section{Principle Design}

Speed - Torque Characteristics Curve. Driving torque of a stepper motor is related with rotation speed and winding current. As shown in the Fig.2, the current of curve 3 is the highest, and that of curve 1 is the lowest. As shown in the figure, the driving torque of stepper motor is inversely proportional to the rotation speed and directly proportional to the winding current, under the same condition. Therefore, since both rotation speed and winding current influence the driving torque, in the application, the speed is fixed firstly and then the current is set to adjust the driving torque.

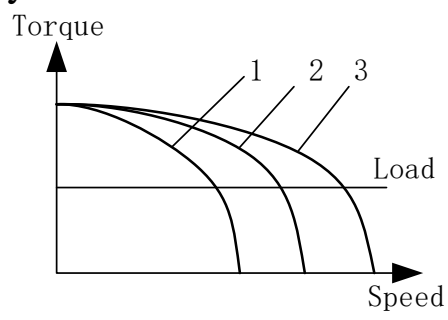

Fig.2 Relationship of driving torque, pulse frequency and winding

Functions Design of the FPGA. This solution is implemented by two fundamental parts : one FPGA and some LMD18200s.

An FPGA, sprouted from PAL, GAL and CPLD, normally consists of CLB (configurable logic block), I/O pads, routing channels and etc. FPGAs are integrated circuit essentially and could be configured to perform complex combination functions. FPGAs have the characteristics of high efficiency, low consumption and strong versatility.

The LMD18200[3], designed by National Semiconductor, is a 3A H-Bridge designed for motion control and compatible with both TTL and CMOS inputs. This device could be used to control the 
winding current by modulating the duty-ratio of PWM signal. Two LMD18200s are needed to drive one two-phase four-wire stepper motor selected in this project.

As shown in Fig.3, the scheme of the FPGA consists of one communication module and some control modules.

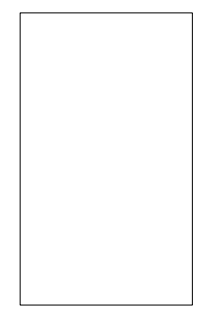

Fig.3 The structure block of the FPGA

The communication module which implements communication function supporting UART communication protocol. By sending instruction packet to the communication module, users set the rotation parameters of stepper motors. As shown in Fig.4,the parameters includes serial number, rotation speed, driving torque, direction, reset and "required position". And the "required position" specifies the number of the steps, that the stepper motor expected to travel after the driver circuit received the hall positioning signal. The controller also sends a status packet to host CPU to provide several information, which may be used for the host. The format of the packet is shown in Fig.4. According to the working status, operators can make the judgment if the resistance has been increased and driving torque is not adequate, then adjusted the duty-ratio of PWM signal. Usually, after 2 or 3 years, the driving torque needs to be changed.

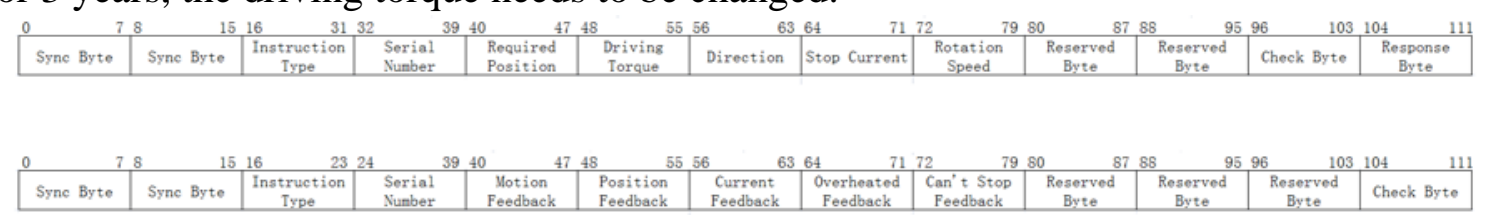

Fig.4 Instruction packet and status packet

Control modules shown in Fig. 3 are designed to control stepper motors. A control module drives a two-phase four-wire stepper motor with two LMD18200s. Control modules could detect the hall positioning signal, operate stepper motors and feed back the status data to the communication module. The control module generates step driving clock according to the time-sequence and required speed. It also generates PWM pulses according the specified duty-ratio. The figure of the driving clock and PWM pulses are shown in Fig.5.

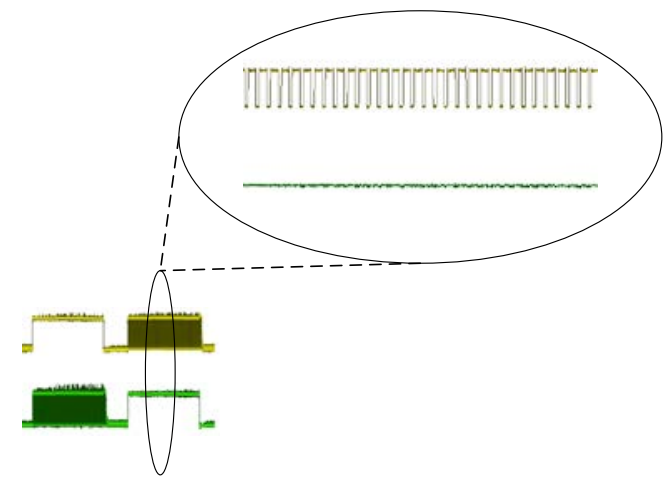

Fig.5 Driving clock and PWM pulses 


\section{Circuit Design}

The block diagram of the system with FPGA as the core of the circuit is shown in Fig.6. Besides the FPGA, the circuit includes four LMD18200s and an UART interface including a DS26LV31T and a DS26LV32AT. The circuit uses an FPGA to receive operation commands from the communication port, output signals to operate stepper motors and feed status data of stepper motors back to the host CPU.

Fig.6 The structure block of the system

In the principle design, the FPGA is Xilinx chip XC3S400-208, which has 400K gates and 120 $\mathrm{I} / \mathrm{Os}$, and is adequate for the usage. The logic design of FPGA is specified using Verilog HDL. The whole scheme is layout on a 4-layer board, and the entity of circuit board is shown in Fig.7. The left connectors are used for output of operation signals and input of hall positioning signal. 4 LMD18200s are installed on the left of the board. The chip in the center of the circuit board is FPGA. On the right edge of board, there are two connectors, one is the power supplies inputs, $+5 \mathrm{~V}$ and $+12 \mathrm{~V}$ DC is required, another one is RS-422 UART interface.

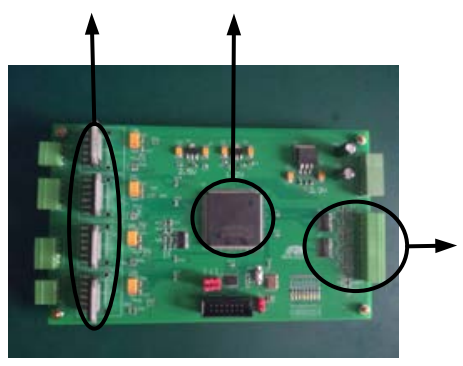

Fig.7 A picture of the circuit

The board shown in Fig.7 is a principle design. The final design installed in satellite will be made with space grade components and the FPAG will be replaced by an Actel anti-fuse device. Also, spare design will be implemented in this device.

\section{Experiment}

To determine how driving torque is adequate, an experiment platform was set up as shown in Fig.8. A stepper motor was placed on the platform and the winding current was adjusted by a PC under the fixed rotation speed. And a current oscilloscope was used to monitor the current. An special mold with known radius was connected to the stepper motor shaft and some weights used to measure the driving torque were connected to the mold. When the weights' suspension point held stably in the plane of the motor shaft, figure out the driving torque with the mass of the weights. By this experimental method, the torque range available by adjusting winding current under the speed can be measured. 


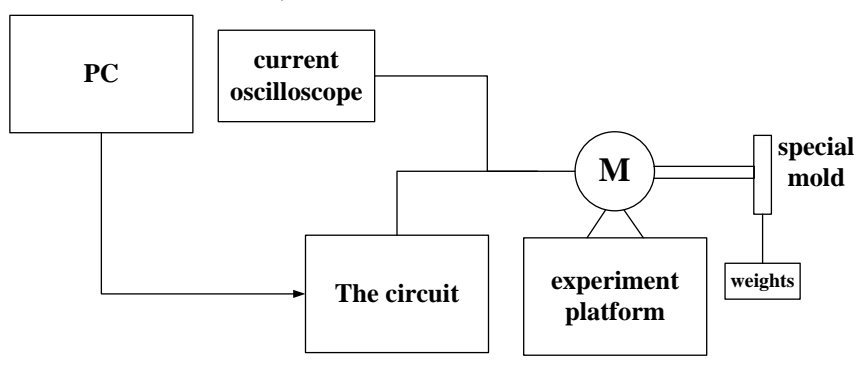

Fig.8 experiment platform

The initial rotation speed is set to 0.8 circle/round, and the initial period of a PWM pulse is 3.2us. Under the initial speed, one step contains 312.5 periods and the stepper motor stars when the duty-ratio of PWM reaches $25 \%$.

Under the initial speed, the relationship of the duty-ratio of PWM signal, the winding current and the driving torque is shown in Fig.9. Fig.9 (a) shows that the winding current changes due to the change of the duty-ratio, and (b) shows the driving torque changes with the current.

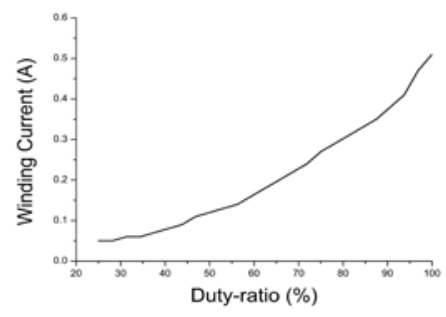

(a)

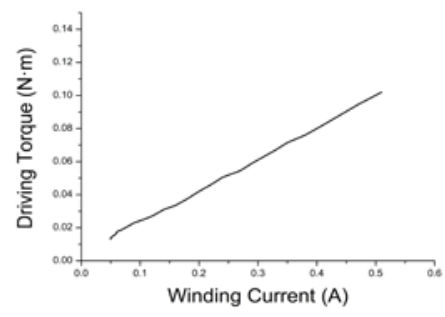

(b)

Fig.9 Relationship of duty-ratio, driving torque and winding current

As the experimental data shows, under the initial rotation speed, the winding current will increase 0.01-0.04A for every $3,125 \%$ increase of the duty-ratio. The adjustment range of driving torque is from $0.013 \mathrm{~N} \cdot \mathrm{m}$ to $0.102 \mathrm{~N} \bullet \mathrm{m}$ and it meets the requirements of the satellite-born spectrometer [4,5].

Furthermore, when the stepper motor runs, the induced electromotive force(EMF) are generated by the changes of the magnetic field generated by the winding current. This kind of induced EMF are also called back electromotive force(BEMF), and the BEMF protects the motor from excessive current and reduces the power consumption of the motor.

The value of BEMF is calculated according to the formula (1).

$$
u=L \frac{d i}{d t}
$$

Where $u$ - induced EMF, $L$ - inductance coefficient, $i$ - winding current, $t$ - time.

As shown in Fig.10, the waveform in big figure is the variation trend of winding current, the small figure is the comparison of the winding current and PWM signal after the amplification of one point on the waveform. The waveform presents a serrated change of winding current with the PWM signal. According to the formula (1), As for driving mode with stable voltage, the driving mode with PWM signal will generate greater BEMF which will more effectively reduce the power consumption of the stepper motor. 


\section{Conclusion}

This paper proposes a design of stepper motors, which applies PMW to adjust the driving torque.

The design has been commissioned and tested. Its functions and characteristics have been verified. The device will be installed in a satellite.

This design is able to be reused in other different applications, which needs an adjustable speed and torque control.

This design uses FPGA to realize the control of stepper motors and generate PWM pulses.

\section{Acknowledgements}

This work was financially supported by the Anhui Provincial Natural Science Foundation (1408085MKL49) and Nation Natural Science Foundation of China.(41275037).

\section{References}

[1] Yu Wang, Yihuai Lu, Xin Zhao, et al: Laser \& Infrared,2015(06): 663-668.(in Chinese)

[2] Jianghong Ke, Fuqi Si, Yibin Fu, et al: Journal of Atmospheric and Environment Optics, 2016,11(2): 103-110. (in Chinese)

[3] Information on http://oap.sourceforge.net/datasheets/AN-694.pdf.

[4] ECSS-E-ST-33-01C, European Cooperation on Space Standardization.

[5] Liehua Zheng, Dayi Yin and Xin Feng: Infrared Technology, 2007(01): 17-21. (in Chinese) 